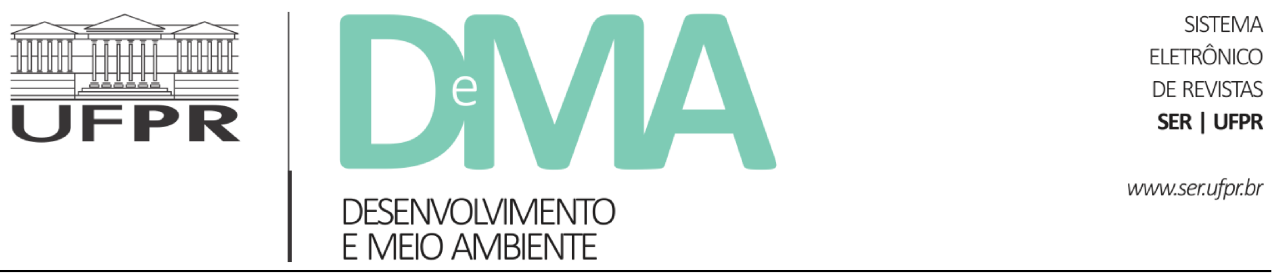

\title{
Pobreza y medio ambiente: una medición con micro-datos para el Norte Grande Argentino
}

\section{Pobreza e meio ambiente: uma medição com microdados para o Norte Grande Argentino}

\section{Poverty and environment: a measurement with microdata for Norte Grande Argentino}

\author{
Fernando Antonio Ignacio GONZÁLEZ ${ }^{*}$, Silvia LONDON ${ }^{1,2}$, Maria Emma SANTOS ${ }^{1,2}$ \\ ${ }^{1}$ Instituto de Investigaciones Económicas y Sociales del Sur, Universidad Nacional del Sur-CONICET, Bahía Blanca, Argentina. \\ ${ }^{2}$ Departamento de Economía, Universidad Nacional del Sur, Bahía Blanca, Argentina.
}

*E-mail de contacto: fernando_gonzalez01@hotmail.com

Artículo recibido el 5 de julio, 2018, versión final aceptada el 27 de febrero, 2019.

RESUMEN: El presente trabajo intenta analizar los vínculos existentes entre pobreza urbana y medio ambiente, con especial énfasis para el caso del Norte Grande Argentino en el período 2011-2016. De esta forma, se analizan las condiciones ambientales que experimentan personas pobres y su situación en relación a los no-pobres, utilizando indicadores relativos a condiciones sanitarias de los hogares, localización de la vivienda, y fuentes de energía empleadas. En todos los casos con datos provistos por la Encuesta Permanente de Hogares publicada por el Instituto Nacional de Estadísticas y Censos. Se encuentra que las personas pobres se enfrentan a un medio ambiente más deteriorado que las no-pobres y que esta situación se agrava en el Norte Grande Argentino en relación al resto del país.

Palabras-claves: pobreza; medio ambiente; Norte Grande Argentino.

RESUMO: O presente trabalho intenta analisar os vínculos existentes entre pobreza urbana e meio ambiente, com especial ênfase para o caso da Região do Norte Grande Argentino (NGA, a partir de agora) no período 2011-2016. Dessa forma, analisam-se as condições ambientais vivenciadas pelos pobres e sua situação em relação aos não pobres, por meio de indicadores relacionados às condições sanitárias domiciliares, à 
localização das moradias e às fontes de energia utilizadas. Em todos os casos com dados fornecidos pelo Inquérito Domiciliar Permanente publicado pelo Instituto Nacional de Estatística e Censos. Os resultados do estudo sugerem que os pobres enfrentam um ambiente mais deteriorado do que os não pobres e que esta situação se agrava no NGA em relação ao resto do país.

Palavras-chave: pobreza; meio ambiente; Norte Grande Argentino.

ABSTRACT: This paper attempts to analyze the links between urban poverty and environment, with special emphasis for the case of the Norte Grande Argentino in the 2011-2016 period. For this purpose, the environmental conditions experienced by poor people and their situation in relation to the non-poor are analyzed, using indicators related to household sanitary conditions, housing location, and sources of energy used. In all cases with data provided by the Permanent Household Survey published by the National Institute of Statistics and Census. The results indicate that poor face a more deteriorated environment than the non-poor and that this situation worsens in the Norte Grande Argentino in relation to the rest of the country.

Keywords: poverty; environment; Norte Grande Argentino.

\section{Introducción}

En la actualidad numerosos países en desarrollo experimentan problemas medioambientales que han ocasionado pérdidas en el ambiente, en salud y económicas para los hogares (Penttinen, 2008). Este fenómeno es comúnmente denominado "degradación ambiental", entendido como el deterioro del ambiente debido al agotamiento de recursos como el suelo, agua, destrucción de los ecosistemas o extinción de la fauna silvestre (Johnson et al., 1997). Y dado que la degradación ambiental aparece relacionada con una alta incidencia de la pobreza, se podría pensar que ambos fenómenos se encuentran causalmente vinculados (Getahun et al., 2018).

De esta forma, en los últimos años se ha ido incrementando la aceptación acerca de la significativa influencia que posee el ambiente sobre el desarrollo humano, especialmente sobre los resultados en términos de salud, conformando lo que se conoce como salud ambiental (Organización Mundial de la
Salud, 2006; Bleich et al., 2012; Galvao et al., 2016). Frecuentemente, dos grandes categorías son identificadas en este caso: padecimientos relacionados a la pobreza (falta de agua potable, sanidad inadecuada en la vivienda y polución dentro del hogar, entre otros) y padecimientos urbanos modernos (polución del aire en las ciudades, exposición a agroquímicos etc.) (Banco Mundial, 2000). Por su parte, los Objetivos de Desarrollo Sostenible (Naciones Unidas, 2015) reflejando la importancia del ambiente en el desarrollo proponen, además de, poner fin a la pobreza en todas sus formas (ODS 1), garantizar la disponibilidad de agua y su gestión sostenible y saneamiento para todos (ODS 6) y garantizar el acceso a una energía asequible, segura, sostenible y moderna para todos (ODS 7).

En este sentido, numerosos estudios sugieren que las personas de menores ingresos son más propensas a sufrir enfermedades infecciosas y tienen mayor mortalidad y morbilidad (Bradley \& Kelleher, 1993), además que soportan mayores riesgos 
físicos y químicos (Hardoy \& Satterthwaite, 2001). Más aún, la evidencia sugiere que los canales más importantes a través de los cuales las condiciones ambientales tendrían un impacto negativo sobre la salud de las personas en situación de pobreza, son la polución del agua y del aire dentro del hogar (Gwatkin \& Guillot, 1999; Satterthwaite, 2003).

De esta manera, se tiende a reconocer la importancia de las características de la vivienda y el vecindario en las interacciones entre pobreza y medio ambiente, examinando dimensiones como: ingresos, acceso a la vivienda, acceso a una provisión de agua mejorada, sanidad, drenaje y recolección de residuos $\mathrm{y}$ acceso a asistencia médica y de emergencias, entre otras (Satterthwaite, 2003; Pasanen et al., 2017).

En este punto, debe entenderse que, si bien la mayoría de los estudios tiende a transmitir la existencia de una relación significativa entre pobreza y condiciones ambientales, no está igualmente claro el sentido de esta relación. Por un lado, se sostiene que los pobres son los causantes de la degradación ambiental y que cualquier política medioambiental debería considerar la mitigación de la pobreza para ser efectiva. Más aún, las personas en situación de pobreza se encontrarían inmersas en un círculo vicioso donde provocan la degradación del ambiente, lo cual a su vez tiende a agravar su situación de escasez de recursos (Comisión Mundial sobre Medio Ambiente y Desarrollo, 1987; Ambler, 1999; Clarke, 1999; Getahun et al., 2018). Contrariamente, se detecta evidencia argumentando que los pobres son empujados a través del mercado a habitar las zonas más degradadas de la ciudad y que su impacto sobre el ambiente es menor que el de los no pobres
(Sunkel, 1980; Satterthwaite, 2003; Pereira \& Nunes da Silva, 2011).

A partir de lo anteriormente dicho, el presente trabajo intenta analizar las interacciones entre pobreza y medio ambiente para el caso particular de la región del Norte Grande Argentino ${ }^{1}$ en el período 2011-2016, considerando especialmente las condiciones habitacionales de los hogares. El análisis de la pobreza y condiciones ambientales resulta especialmente relevante en una región como el NGA, que históricamente ha evidenciado un menor grado de desarrollo económico y humano en relación al resto del país. Esto puede ser observado en la brecha existente con el resto de la Argentina, en términos de pobreza monetaria, necesidades básicas insatisfechas (NBI), mortalidad infantil, desnutrición, analfabetismo o acceso a servicios públicos, entre otros (Bolsi et al., 2005; Longhi \& Osatinsky, 2017).

En lo que sigue, la sección 2 presenta los principales antecedentes en el tema. La sección 3 describe algunos indicadores y metodología utilizados en la evidencia previa, mientras que la sección 4 presenta los indicadores y bases de datos a emplear. Finalmente, las secciones 5 y 6 presentan los principales resultados y conclusiones, respectivamente.

\section{Nexo Pobreza-Medio Ambiente}

Entendiendo que pobreza no se refiere únicamente a la falta de ingresos monetarios, sino también a la existencia de múltiples privaciones simultáneas en términos de educación, acceso a cobertura de salud, vivienda adecuada, alimentación etc., (Alkire \& Santos, 2010; Santos 2014) es posible comprender

${ }^{1}$ Compuesto por las provincias de: Misiones, Corrientes, Chacho, Formosa, Jujuy, Catamarca, La Rioja, Tucumán, Santiago del Estero y Salta. 
en forma más directa los vínculos existentes entre este fenómeno y el medio ambiente.

Frecuentemente, se afirma que las personas en situación de pobreza emplean sus recursos en asegurar su subsistencia sin considerar el ahorro para consumo futuro o conservación ambiental (Prakash, 1997). Se comportarían como maximizadores con un horizonte temporal de corto plazo, tratando de satisfacer sus necesidades presentes, a costa de beneficios futuros. De esta forma, numerosa evidencia tiende a avalar la idea que las personas pobres sobreexplotan los recursos a su alcance, contribuyendo a la degradación ambiental (Comisión Mundial sobre Medio Ambiente y Desarrollo, 1987; Nayak, 2004; Getahun et al., 2018).

En este sentido, es ampliamente estudiada la relación existente entre deforestación y pobreza, donde se detecta que las personas pobres tienden a sobre-explotar los recursos forestales en usos como combustible, forraje o materiales de construcción (Vedeld et al., 2007). Este fenómeno es especialmente relevante para algunas provincias argentinas como Misiones que cuenta con abundantes bosques nativos (Brown, 2009), los cuales constituyen una de las mayores fuentes de biodiversidad del planeta (Olson \& Dinerstein, 2002) y han perdido una porción considerable de su superficie original (Mittermeir et al., 2005). En la misma dirección, se han relacionado con la pobreza otras fuentes de degradación ambiental: erosión del suelo o agotamiento de nutrientes (Sherr \& Yadav, 1997), contaminación de los cursos de agua (Cernea, 1990), hacinamiento urbano (Nayak, 2004), entre otros.

De esta forma, implícitamente se vincula la noción de riqueza y crecimiento con mejoras en el ambiente; sin embargo, esta idea no es exclusiva de la literatura económica, sino que es compartida con otros campos como la psicología y la sociología, con conceptos como la de jerarquía de necesidades: una vez que una persona satisface sus necesidades básicas, podría empezar a considerar necesidades de orden superior, incluyendo la conservación y cuidado ambiental (Gertsakis \& Lewis, 2003).

Complementando 1o anterior, se detecta evidencia empírica consistente con la idea que la degradación ambiental, a su vez, tiende a empeorar la situación de las personas pobres, dando lugar a la existencia de un círculo vicioso o espiral descendente (Gray \& Moseley, 2005). De esta forma, la degradación ambiental implicaría una reducción en la base de recursos de las personas pobres, lo que los volvería menos productivos, más susceptibles a eventos extremos y en última instancia, agravaría su situación de pobreza (Sunkel, 1980; Comisión Mundial sobre Medio Ambiente y Desarrollo, 1987; Moore, 2001). Estos estudios sugieren que las personas pobres son más vulnerables a la pérdida de recursos naturales dado que estos representan una mayor proporción de sus ingresos (Banco Mundial, 2005). Además, la degradación ambiental reduciría la capacidad de las personas pobres de generar ingresos, requiriéndoles más tiempo en tareas domésticas rutinarias (Mink, 1993).

Considerando aspectos de salud, al contar con peores sistemas de acceso a agua segura, viviendas inadecuadas, precarios sistemas de disposición de residuos etc., las personas pobres también serían más propensas a padecer enfermedades como dengue o leishmaniasis (Organización Mundial de la Salud, 2004), además de enfermedades respiratorias provocadas por la polución del aire dentro del hogar (Bucknall et al., 2001). Esto resulta relevante para las provincias del NGA que tienden a presentar una elevada incidencia de casos de dengue y fiebre amarilla (Sociedad Entomológica Argentina, 2009). 
Por otro lado, en disonancia con lo anterior, se observa evidencia contraria a la idea de la pobreza como responsable de la degradación ambiental (Satterthwaite, 2003; Pereira \& Nunes Da Silva, 2011). Se argumenta que, en realidad, las personas marginadas socialmente (como aproximación a una situación de pobreza), son aún más marginadas al estar localizadas en zonas ecológicas marginales; más aún, en algunos casos se observa que, dadas las industrias contaminantes instaladas frecuentemente en vecindarios urbanos pobres, éstos tendrían un mayor interés en la problemática ambiental considerando que se verían afectados en forma directa (Kurtz, 2003). En este sentido, el riesgo ambiental contribuiría a generar pobreza urbana debido a mayores costos económicos por enfermedades, mientras que los pobres no contribuirían a la degradación ambiental en mayor medida que los no pobres, asociando el fenómeno de la degradación a los patrones de consumo de la clase media y alta y a la falla del gobierno en implementar políticas ambientales efectivas (Satterthwaite, 2003).

Finalmente, algunos estudios encuentran que las condiciones ambientales que enfrentan las personas en situación de pobreza no es significativamente distinta de aquellas no-pobres (Carter et al., 2007). Esto implicaría que, además de no existir consenso acerca de la direccionalidad del nexo pobreza-ambiente, tampoco es clara la existencia de tal relación.

\section{Hacia una operacionalización del Nexo Pobreza-Medio Ambiente}

En general, las investigaciones sobre el nexo pobreza-medio ambiente, consideran diferentes indicadores de acuerdo a restricciones en los datos disponibles. Se observa cierta regularidad en la utilización de microdatos a nivel de hogar (Okwi et al., 2006; Pasanen et al., 2017), los cuales, en algunos casos, son complementados con macrodatos (Bhattacarya \& Innes, 2006).

En este sentido, Satterthwaite (2003) propone un conjunto de indicadores para analizar pobreza y su interacción con el medio ambiente en contextos urbanos: Ingresos y endeudamiento, base de activos, vivienda (materiales, calidad del aire interior, etc.), provisión de infraestructura pública (agua, cloacas, caminos, gestión de residuos etc.), provisión de servicios básicos (cuidados médicos, escuelas, cumplimiento de la ley, transporte, comunicaciones, etc.), redes de seguridad para asegurar una alimentación básica, protección de los más pobres (contra la violencia, discriminación o explotación) y finalmente, voz y poder de los pobres (en el sistema político y la estructura burocrática). Se afirma que la evolución de estos indicadores debería ser monitoreada periódicamente para comprender las interacciones existentes.

Por su parte, Clichevsky (2002), afirma que los principales problemas urbano-ambientales que afectan a los pobres, y que deben ser considerados al analizar el nexo pobreza-ambiente, son: un sistema de agua potable con calidad insuficiente o inexistencia del mismo, inadecuada provisión de cloacas, falta de un sistema integral de recolección y disposición de residuos domiciliarios, contaminación de los cursos de agua urbanos y el alto grado de hacinamiento y precariedad laboral. Concordantemente, Pereira \& Nunes da Silva (2011) argumentan que deben ser consideradas las limitaciones impuestas por el mercado inmobiliario a la periferia urbana en términos de limitaciones en los servicios urbanos 
básicos, situación sanitaria, viviendas inadecuadas o riesgo de inundaciones.

Enfocándose en la cuestión particular de los indicadores de pobreza-medio ambiente y su aplicación en países de África, Twesigye (2007), considera necesaria la inclusión de cuatro grandes categorías de indicadores: pobreza-recursos naturales (que cuantifique la dependencia de los pobres sobre los recursos naturales), pobreza-salud ambiental (vulnerabilidad generada por polución del aire o agua), pobreza-vulnerabilidad a desastres ambientales (medida de la exposición de los pobres a desastres naturales como inundaciones o sequías y sus costos asociados) y pobreza-vivienda (indicadores de las condiciones habitacionales de los pobres y como afecta su situación de pobreza).

Por su parte, la World Wild Fund (2004) propone una serie de tres categorías para evaluar las interacciones entre pobreza y medio ambiente en países en desarrollo: Indicadores de status: (porcentaje de hogares con agua segura, porcentaje de hogares con tenencia legal de su hogar, cantidad de hectáreas forestadas, entre otros), Indicadores de condiciones aptas (se incluye la existencia de instituciones que fomenten el estudio del nexo pobreza-ambiente, marco legal sobre cuidado del medio ambiente, empoderamiento y participación de los pobres, porcentaje del presupuesto destinado a pobreza y ambiente, etc.) e Indicadores de capital social.

En síntesis, se observa cierta regularidad en incluir indicadores de vivienda, dependencia de los recursos naturales, acceso a servicios básicos, riesgo de desastre ambiental y capital social, al analizar las interacciones presentes entre pobreza y ambiente. Naturalmente, surge el interrogante de cómo aplicar estos indicadores para evaluar las interacciones. Avanzando en este tópico, Dasgupta et al. (2005) argumentan que contando con datos a nivel de núcleos poblacionales es posible analizar si los problemas ambientales afectan desproporcionadamente a aquellas regiones con una mayor intensidad de la pobreza. De esta forma, Dasgupta et al. (2005) lleva a cabo análisis de regresión para estimar la significatividad de relaciones como pobreza-deforestación, pobreza-uso de leña. Además, se evalúa el acceso a agua salubre y cloacas, comparando entre regiones. En el mismo sentido avanzan Aggrey et al. (2010), sosteniendo que en el caso de detectar que aquellas regiones con mayor intensidad de pobreza, también presentan un medio ambiente más degradado, se verificaría la existencia de un nexo entre ambos fenómenos.

Profundizando, Nussbaumer et al. (2012) construyen un índice multidimensional, a partir de la metodología Alkire-Foster, vinculando pobreza y ambiente (específicamente, consumo de energía). De esta forma, trabajando con datos a nivel de hogar, utilizan indicadores como combustible utilizado para cocinar, acceso a electricidad o tenencia de heladera que lo combinan en un único índice compuesto.

Por su parte, Narloch \& Bangalore (2018), utilizan datos a nivel de hogar y de región. Realizan análisis de regresión y correlación y encuentran que las regiones más pobres presentan peores condiciones ambientales, a la vez que, dentro de una misma región, los hogares pobres se encuentran peor posicionados que sus pares no-pobres. Finalmente, encuentran que las condiciones ambientales explican una parte significativa de las diferencias en el consumo de los hogares, pero no así, de los cambios en el tiempo. 
Finalmente, Thiry et al. (2018), proponen incorporar la dimensión ambiental al análisis de la pobreza y afirman que es posible complementar a los microdatos de hogar, con indicadores ambientales a nivel local o regional. Aunque aclaran que se perdería, en este caso, variabilidad entre hogares

\section{Metodología}

Dado el objetivo de evaluar, exploratoriamente, la forma en que se presenta la interacción entre pobreza y medio ambiente para el caso del Norte Grande Argentino, es que se opta por usar la Encuesta Permanente de Hogares (EPH) para el período 2011-2016. ${ }^{2}$ Esta base de datos presenta la ventaja de tener una alta periodicidad (trimestral) e incluye datos desagregados por provincia.

De esta forma, se puede comparar los diferentes contextos a los que son sometidas personas pobres y no podres, a partir del trabajo con micro-datos. En este punto se debe tener en cuenta, las limitaciones en la disponibilidad de datos en la EPH: solo contempla áreas urbanas y no incluye algunas de las variables que se detectan relevantes para el análisis, como indicadores directos del capital social o acceso a electricidad residencial.

En este sentido, y teniendo en cuenta que las investigaciones previas tienden a apoyarse ampliamente en tres categorías: condiciones habitacionales del hogar, condiciones del entorno cercano (barrio o vecindario) e instituciones, es que se propone considerar los siguientes indicadores para evaluar la interacción entre pobreza y medio ambiente en el caso del NGA:
1. Tasa de dependencia efectiva elevada: hogares con más de 2 miembros inactivos por cada persona ocupada.

2. Tenencia precaria de la vivienda: vivienda ocupada ilegalmente $\mathrm{u}$ ocupada en forma gratuita $\sin$ contrato.

3. Hacinamiento: hogares con 3 o más personas por cuarto o sin cuarto alguno.

4. Consumo de agua en condiciones precarias: hogares sin acceso a agua dentro de la vivienda, o agua proveniente de pozo.

5. Sistema de disposición de excretas inadecuado: hogares que no tienen baño, o que el inodoro no posee mochila/cadena, o el desagüe del baño es a hoyo en el suelo o a pozo ciego sin cámara séptica.

6. Combustible usado para cocinar perjudicial: hogares que utilizan kerosene, leña o carbón para cocinar dentro del hogar.

7. Riesgo inundación: localización de la vivienda en áreas inundables

8. Riesgo sanitario: localización de la vivienda a menos de 300 metros de un basural.

Conjuntamente, dada la forma en que se definen los indicadores planteados anteriormente, es posible analizar las carencias que enfrenta cada hogar a partir del cumplimiento o no de los estándares mínimos propuestos para cada indicador. Al emplear variables binarias (se presenta carencia o no), es plausible estandarizar los resultados para facilitar la comparación, otorgándole a cada indicador el valor 1 si la persona no alcanza el estándar mínimo propuesto y el valor 0 , en otro caso. Esto es consistente, en forma parcial, con la metodología de medición de pobreza multidimensional Alkire-Foster (Alkire \& Foster, 2007; 2011). 
En este trabajo no se avanza en la etapa de agregación de los resultados y, por ende, no se propone un índice compuesto.

Finalmente, a la hora de identificar a aquellas personas pobres, se sigue el método indirecto de pobreza por ingresos; es decir, todas aquellas personas que no alcancen un ingreso monetario (considerando la cantidad de personas en el hogar, sexo y edad) suficiente para cubrir el costo de una canasta básica ${ }^{3}$ que le permita satisfacer necesidades alimenticias y no alimenticias, serán consideradas pobres. No deben dejar de ser resaltadas las amplias limitaciones de este método, unidimensional e indirecto, además del creciente consenso de la insuficiencia de los ingresos como indicador de bienestar.

\section{Resultados}

\subsection{Pobreza por ingresos}

En primer lugar, se presentan los resultados de las estimaciones de pobreza por ingresos para el NGA y el total de la Argentina urbana (Tabla 1).

TABLA 1 - Proporción de personas pobres en el NGA y Argentina, 2011-2016.

\begin{tabular}{lll}
\hline Año & NGA & Argentina \\
\hline 2011 & 37,44 & 27,27 \\
\hline 2012 & 34,99 & 25,99 \\
\hline 2013 & 33,27 & 24,22 \\
\hline 2014 & 37,94 & 28,81 \\
\hline 2015 & 32,76 & 24,71 \\
\hline 2016 & 32,26 & 25,89 \\
\hline
\end{tabular}

FUENTE: elaboración propia en base a datos de EPH.
De los datos anteriores pueden ser extraídos dos resultados: en primer lugar, el NGA presenta mayores niveles de pobreza que el promedio nacional (NGA con 33\% más de personas pobres en todo el período), algo que a priori era esperable. En segundo lugar, la brecha de pobreza entre en NGA y Argentina se reduce levemente hacia 2016 (NGA con $24 \%$ más de personas pobres).

\subsubsection{Perfiles de pobreza}

Resulta relevante no solamente conocer la proporción de personas u hogares en situación de pobreza en un momento de tiempo o región, sino también las características que presentan estos núcleos familiares tales como nivel educativo de sus integrantes, localización, cantidad de miembros, condición de actividad, etc. y que en definitiva conformarán lo que se denomina "perfiles de pobreza".

La Tabla 2 presenta información relevante para construir estos perfiles donde han sido seleccionados aquellos que representan a una mayor proporción de personas pobres y que se encuentran más sobredimensionados en términos de pobreza.

En base a los datos, es posible afirmar que en promedio un hogar en situación de pobreza monetaria tiende a presentar las siguientes características con mayor probabilidad que las correspondientes alternativas de cada caso: se sitúan en el Norte Grande Argentino, el jefe de hogar no terminó el colegio secundario, se encuentra trabajando en relación de dependencia, pero en condiciones informales y el hogar presenta una gran proporción de personas inactivas por cada persona ocupada $(>2)$. A su vez,

${ }^{3}$ Dada la subestimación de la inflación por parte del INDEC entre 2007-2015, se empleará la canasta básica elaborada por la Fundación de Investigaciones Económicas Latinoamericanas (FIEL) entre 2011-2016. 
TABLA 2 - Perfil de pobreza en Argentina, 2011-2016.

\begin{tabular}{lllll}
\hline Grupo Vulnerable & $\begin{array}{l}\text { Proporción de } \\
\text { miembros del } \\
\text { grupo que son } \\
\text { pobres }\end{array}$ & $\begin{array}{l}\text { Proporción } \\
\text { de pobres que } \\
\text { pertenecen a } \\
\text { grupo (A) }\end{array}$ & $\begin{array}{l}\text { Proporción de } \\
\text { la población } \\
\text { que pertenece al } \\
\text { grupo (B) }\end{array}$ & $\begin{array}{l}\text { Grado de sobre } \\
\text { representación } \\
\text { entre los pobres } \\
\text { (A/B) }\end{array}$ \\
\hline NGA & 34,13 & 42,38 & 31,35 & 1,35 \\
\hline Jefe de Hogar Mujer & 29,86 & 37,07 & 32,99 & 1,12 \\
\hline Jefe de Hogar sin secundario completo & 36,01 & 73 & 53,88 & 1,35 \\
\hline Jefe de Hogar desempleado & 60,14 & 6,09 & 2,69 & 2,26 \\
\hline Jefe de Hogar empleado informal & 47,33 & 26,6 & 14,94 & 1,78 \\
\hline Tasa de Dependencia Efectiva Elevada & 45,61 & 64,68 & 37,69 & 1,72 \\
\hline
\end{tabular}

FUENTE: elaboración propia en base a datos de Encuesta Permanente de Hogares.

se puede observar que el hecho que el hogar esté encabezado por una mujer incrementa sus chances de estar en situación de pobreza, al igual que un hogar con un jefe de hogar desempleado.

\subsection{Privaciones ambientales $(P A)$}

A continuación, se presentan, en forma gráfica, los resultados de Condiciones Ambientales (Figura 1).

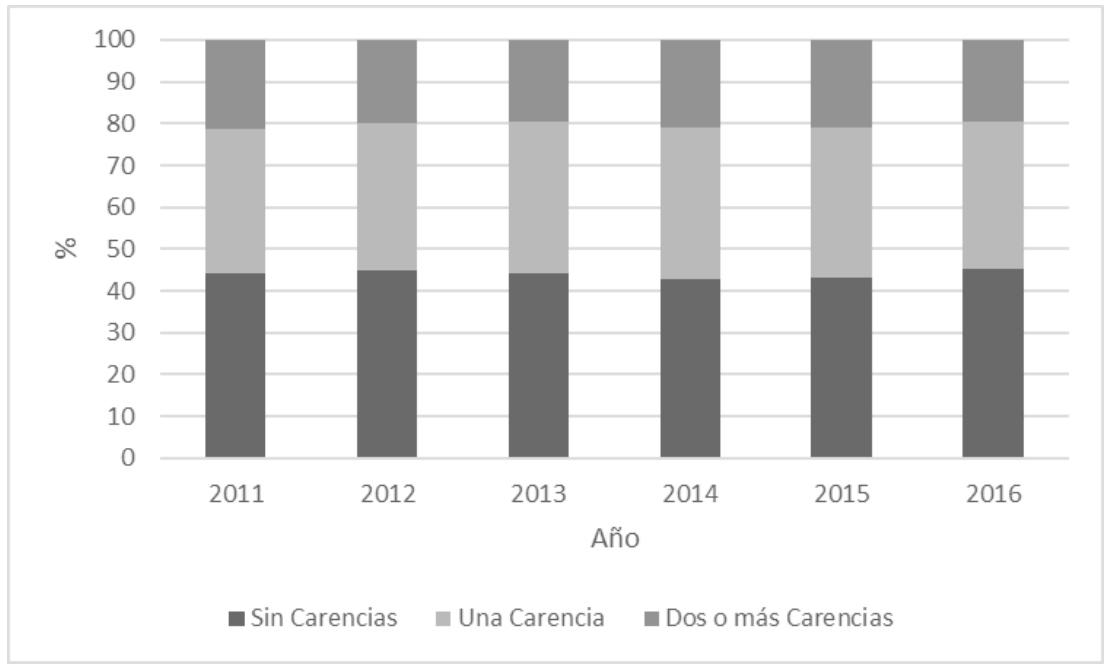

FIGURA 1 - Privaciones ambientales en Argentina.

FUENTE: elaboración propia en base a datos de Encuesta Permanente de Hogares. 
Se puede observar de la Figura 1 que, la mayor parte de las personas no presenta privación alguna en los indicadores seleccionados (44\%) o, a lo sumo, presenta una carencia (35\%). En consonancia con lo anterior, se detecta que la proporción de personas sin carencias, tiende a incrementarse levemente $(+1 \mathrm{pp})$, al mismo tiempo que el porcentaje de personas con 2 o más privaciones disminuye (-2 pp). Estos resultados indicarían que además de disminuir la proporción de personas que experimentan privaciones (al menos una), también disminuyó la intensidad experimentada (la cantidad de privaciones simultáneas).

En el caso del NGA, se observan tendencias similares. Con aumento de la proporción de personas sin privaciones $(+5 \mathrm{pp})$; de este modo, si bien en 2011 el NGA partía con un $37 \%$ de personas sin carencias (versus $44 \%$ a nivel nacional), se arriba a 2016 , con $42 \%$ de personas sin carencias (versus $45 \%$ a nivel nacional) lo que evidenciaría una mejora significativa del NGA en términos absolutos y relativos.

La tendencia a una igualación en las condiciones ambientales entre el NGA y el resto de los aglomerados urbanos incluidos en la EPH, es consistente con evidencia empírica anterior elaborada en materia de pobreza multidimensional, donde se emplean algunos indicadores coincidentes con los aquí presentados (Arevalo \& Paz, 2015). Sin embargo, para poder afirmar inequívocamente que las condiciones ambientales en alguna región son mejores o peores que las de otra, sin importar la cantidad de privaciones que se elija para la comparación, cabría realizar un análisis de dominancia estocástica (Figuras 3 y 4).

Se observan las funciones de distribución acumulada para cada caso, en 2011 y 2016, donde la función del NGA siempre se encuentra por debajo, o al mismo nivel, que la función de Argentina. A partir de 7 privaciones, ambas funciones alcanzan

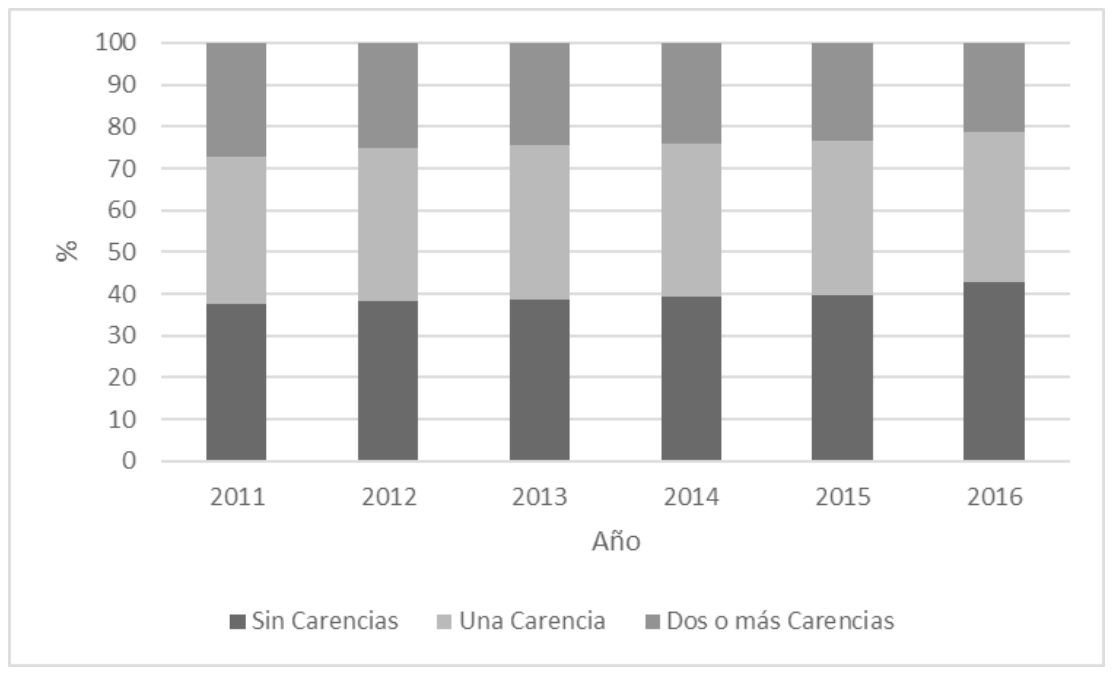

FIGURA 2 - Privaciones ambientales en el Norte Grande Argentino.

FUENTE: elaboración propia en base a datos de Encuesta Permanente de Hogares. 
el $100 \%$. Este comportamiento de las curvas, que en ningún punto se cruzan, indica la existencia de dominancia estocástica de primer orden del NGA sobre Argentina y por ello, es posible afirmar que las condiciones ambientales son inequívocamente peores en el NGA que en el resto del país; aunque se debe tener presente que la distancia entre ambas distribuciones tiende a reducirse con el paso del tiempo tal como se observa en las figuras.

El análisis puede ser complementado con algún test formal para asegurar que las diferencias observadas gráficamente son significativas estadísticamente.

En este sentido, se emplea el test desarrollado en Santos \& Zaratiegui (2017) donde se utiliza una prueba del tipo Kolmogorov-Smirnov para comparar dos distribuciones. De este modo, para los años analizados, empleando un valor crítico de 1,2239 y un nivel de significancia del $5 \%$, se rechaza la hipótesis nula que la función de distribución acumulada (FDA) de Argentina domine estocásticamente a la FDA del NGA y no se rechaza la hipótesis nula que la FDA del NGA domine estocásticamente en primer orden a la FDA de Argentina; por ende, cabe concluir que las condiciones ambientales son inequívocamente peores en el NGA que en el resto del país.

TABLA 3 - Valor crítico y estadísticos, años 2011 y 2016.

\begin{tabular}{lcc}
\hline & $\mathbf{2 0 1 1}$ & $\mathbf{2 0 1 6}$ \\
\hline Valor Crítico & 1,2239 & 1,2239 \\
\hline $\begin{array}{l}\text { Estadístico del test (Ho: } \\
\text { NGA domina a ARG) }\end{array}$ & 0 & 0,02 \\
\hline $\begin{array}{l}\text { Estadístico del test (Ho: } \\
\text { ARG domina a NGA) }\end{array}$ & 11,77 & 17,33 \\
\hline
\end{tabular}

FUENTE: elaboración propia en base a datos de Encuesta Permanente de Hogares
Luego de comparar niveles de privaciones, es pertinente analizar qué carencias experimentan las personas y en este sentido, para el año 2016, el perfil de privaciones para el NGA y Argentina es el siguiente (Figuras 5 y 6 ).

De la comparación de las figuras anteriores se puede observar que el NGA tiende a presentar una mayor proporción de personas con carencias en 5 de los 8 indicadores analizados: mientras más se aleje del centro el área sombreada, mayor proporción de personas con carencias en ese indicador. Destacan especialmente en este sentido, los indicadores de tasa de dependencia efectiva (que alcanza al 38\% de las personas del NGA), agua insalubre, combustible perjudicial, riesgo inundación y riesgo sanitario. Por su parte, en el indicador de hacinamiento no se observan diferencias apreciables, mientras que el NGA presenta mejores resultados en los indicadores de tenencia y disposición de excretas inadecuado.

Finalmente, resulta relevante analizar las condiciones ambientales que enfrentan aquellas personas consideradas pobres monetariamente. En este sentido, en el NGA para el año 2016 se presentan los siguientes resultados (Figura 7).

Se puede observar que las personas en situación de pobreza monetaria se enfrentan a condiciones ambientales más adversas que los no pobres. En este sentido, más del $80 \%$ de las personas pobres del NGA experimentan al menos una privación ambiental; este porcentaje es significativamente más elevado que el $50 \%$ de las personas no pobres de la misma región. Conviene tener presente que también en este caso (al igual que en la comparación NGA-Argentina) es posible afirmar inequívocamente que las personas pobres experimentan peores condiciones ambientales 


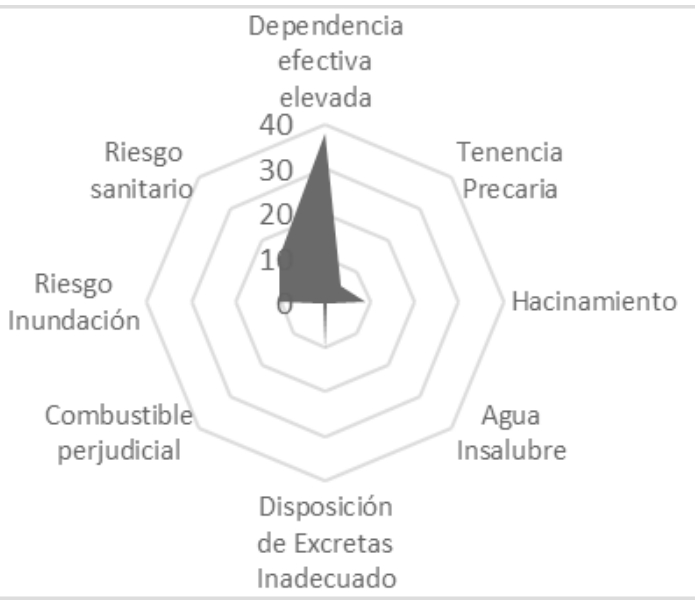

FIGURA 5 - Privaciones por Indicador en Norte Grande Argentino.

FUENTE: elaboración propia en base a datos de Encuesta Permanente de Hogares.

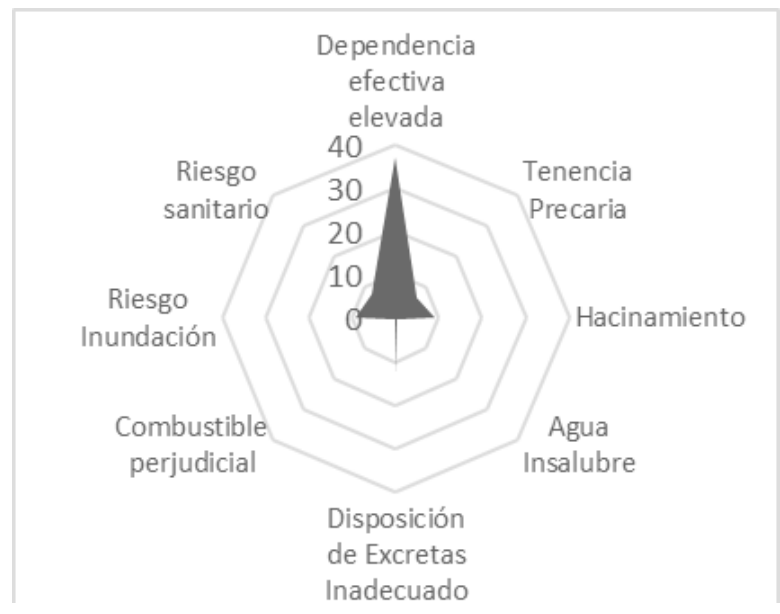

FIGURA 6 - Privaciones por Indicador en Argentina.

FUENTE: elaboración propia en base a datos de Encuesta Permanente de Hogares. que las personas no pobres dada la existencia de dominancia estocástica de primer orden entre las funciones de distribución acumuladas de ambos grupos (Figura 8).

Sabiendo que personas pobres presentan peores condiciones ambientales, es relevante conocer la magnitud de las diferencias para los distintos indicadores. En este sentido se observa lo siguiente (Tabla 4).

TABLA 4 - Porcentaje de privaciones por indicador, en personas pobres y no pobres del Norte Grande Argentino, año 2016.

\begin{tabular}{lll}
\hline Indicador & Pobres & No pobres \\
\hline $\begin{array}{l}\text { Dependencia efectiva } \\
\text { elevada }\end{array}$ & 59,33 & 27,68 \\
\hline Tenencia Precaria & 6,61 & 5,06 \\
\hline Hacinamiento & 19,36 & 4,4 \\
\hline Agua insalubre & 0,63 & 0,33 \\
\hline $\begin{array}{l}\text { Disposición de excretas } \\
\text { inadecuado }\end{array}$ & 17,33 & 7,38 \\
\hline Combustible perjudicial & 0,55 & 0,49 \\
\hline Riesgo inundación & 13,83 & 8,36 \\
\hline Riesgo sanitario & 17,45 & 12,21 \\
\hline
\end{tabular}

FUENTE: elaboración propia en base a datos de Encuesta Permanente de Hogares.

${ }^{4}$ La hipótesis nula será rechazada siempre que el valor del estadístico sea mayor al valor crítico 
Puede ser comprendido nuevamente con estos datos que las personas en situación de pobreza soportan peores condiciones ambientales: para el conjunto de los diez indicadores seleccionados, el resultado de los pobres es peor que el de personas no pobres. Además, la brecha entre ambos grupos es especialmente amplia en algunos indicadores: el hecho de ser pobre, incrementa significativamente el riesgo de sufrir inundaciones, vivir en condiciones de hacinamiento o no tener acceso a agua segura. A pesar de lo anterior, no se observar diferencias significativas en el indicador de Combustible perjudicial.

En último lugar, es relevante considerar las diferencias de condiciones ambientales experimentadas por pobres y no pobres, dentro de una misma región; en este sentido se obtiene (Tabla 5).
TABLA 5 - Cociente de privaciones ambientales entre pobres y no pobres.

\begin{tabular}{lllllll}
\hline & $\mathbf{2 0 1 1}$ & $\mathbf{2 0 1 2}$ & $\mathbf{2 0 1 3}$ & $\mathbf{2 0 1 4}$ & $\mathbf{2 0 1 5}$ & $\mathbf{2 0 1 6}$ \\
\hline NGA & 2,38 & 2,38 & 2,18 & 2,19 & 2,15 & 2,25 \\
\hline Argentina & 2,63 & 2,61 & 2,46 & 2,4 & 2,36 & 2,41 \\
\hline
\end{tabular}

FUENTE: elaboración propia en base a datos de Encuesta Permanente de Hogares.

Estos resultados deben ser interpretados de la siguiente manera: en términos de la cantidad de privaciones, una persona pobre del NGA experimentó en el año 2016 un 125\% más de privaciones que una persona no pobre de la misma región, en promedio. De esta manera, los datos sugieren que la desigualdad presente entre las condiciones ambientales que experimentan pobres y no pobres, es menor en el NGA que en el resto del país.

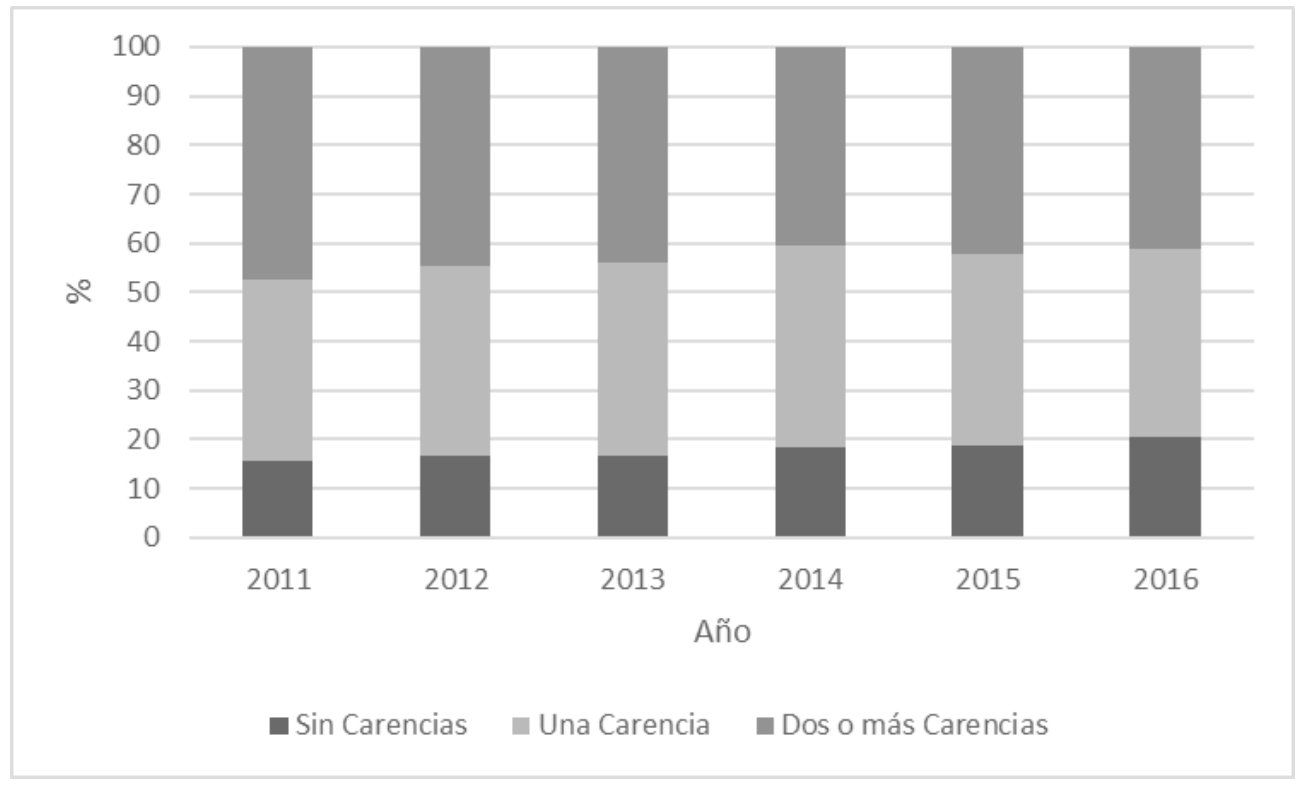

FIGURA 7 - Privaciones ambientales de personas pobres, Norte Grande Argentino 2016.

FUENTE: elaboración propia en base a datos de Encuesta Permanente de Hogares. 


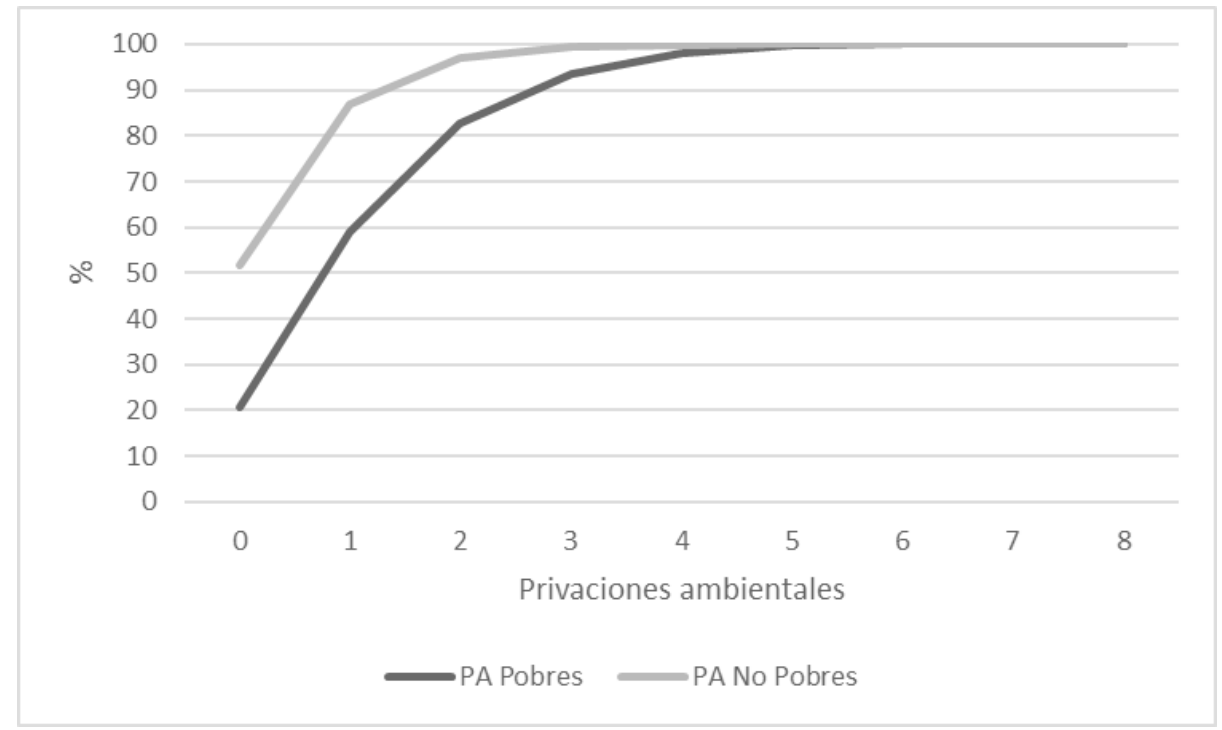

FIGURA 8 - Privaciones ambientales de personas pobres, Norte Grande Argentino 2016.

FUENTE: elaboración propia en base a datos de Encuesta Permanente de Hogares.

\section{Conclusiones}

Se ha analizado el problema de la interrelación entre pobreza y medio ambiente en el contexto de la argentina urbana y especialmente en el Norte Grande Argentino, en el período 2011-2016.

Se encontró que los niveles de pobreza monetaria son más elevados, a lo largo de todos los años analizados, en el NGA que en el resto del país. Aunque con una tendencia a la reducción de las disparidades en el último bienio. Además, las condiciones ambientales, medidas a través de los indicadores aquí propuestos, se presentaron más deterioradas en el NGA que en el resto del país. Este resultado se mostró robusto y estadísticamente significativo.

En segundo lugar, se observó que las personas en situación de pobreza monetaria experimentaron peores condiciones ambientales que las personas no pobres, sin importar la región analizada. Las diferencias son especialmente amplias en los indicadores de acceso a agua segura, hacinamiento y localización en zona inundable. Finalmente, se detectó que la desigualdad, medida por las condiciones ambientales, entre personas pobres y no pobres, es menor en el NGA que en el resto del país. En el mismo orden de ideas, se observó una mayor utilización de combustibles como leña o carbón en los hogares pobres, aunque dicha diferencia es reducida, en comparación a la de otros indicadores.

Por todo lo anterior, es posible afirmar que existe un nexo significativo entre pobreza y medio ambiente en el NGA, lo que es concordante con la mayor parte de la evidencia empírica en el tema. Lo anterior sugiere que el fenómeno de la pobreza debe ser entendido desde una óptica multidimen- 
sional, dada la existencia de múltiples factores socio-ambientales que lo afectan y profundizan, en concordancia con abundante literatura previa (Alkire \& Santos, 2010; Santos, 2014).

A futuro, se considera relevante poder incorporar al análisis, más indicadores que traten de cuantificar la utilización de recursos ambientales en entornos urbanos, a la vez, de incluir macro-datos que presenten diferencias entre aglomerados para profundizar las comparaciones entre regiones.

\section{Referencias}

Aggrey, N.; Wanbugu, S.; Karugia J.; Wanga, E. An Investigation of the poverty-Environment Degradation Nexus: A case study of Katonga Basin in Uganda. Research Journal of Environmental and Earth Sciences, 2(2), 82-88, 2010.

Alkire, S.; Foster, J. Recuento y medición multidimensional de la pobreza. OPHI Working Paper 7, University of Oxford, 2007. Disponível em: https://ophi.org.uk/recuento-y-medicion-multidimensional-de-la-pobreza/

Alkire, S.; Foster, J. Understandings and misunderstandings of multidimensional poverty measurement. OPHI Working Paper 43, University of Oxford, 2011. Disponível em: https://ophi.org.uk/understandings-and-misunderstandings-of-multidimensional-poverty-measurement/

Alkire, S.; Santos M. Acute Multidimensional Poverty: A New Index for Developing Countries. OPHI Working Papers 38, University of Oxford, 2010. Disponível em: https://ophi.org.uk/acute-multidimensional-poverty-a-new-index-for-developing-countries/

Ambler, J. Attacking Poverty While Improving the Environment: Toward Win-Win Policy Options. Nueva York, Ed.UNDP-EC Poverty and Environment Initiative, 1999.

Arevalo, C.; Paz J. Pobreza en Argentina. Privaciones múltiples y asimetrias regionales. Documento de trabajo 15 Ielde-Unas, 2015. Disponível em: http://www.economicas. unsa.edu.ar/ielde/items_upload/WPIelde_Nro_15.pdf
Banco Mundial. Health and Environment. Environment Strategy Background Series, 2000. Disponível em: http://documents.worldbank.org/curated/en/569631468766229873/ pdf/multi0page.pdf. Acesso em: mar. 2017.

Banco Mundial. Ensuring environmental sustainability: measuring progress toward the 7th Millenium Development Goal. Documento de trabajo, 2005. Disponível em: http://documents.worldbank.org/curated/en/969821468332405888/ Ensuring-environmental-sustainability-measuring-progress-toward-the-7th-millennium-development-goal. Acesso em: jun, 2018.

Bhattacharya, H.; Innes, R. Is there a Nexus between poverty and environment in rural India? En: Anales de la Reunión Anual de la Asociación Americana de Economía Agrícola, California 23-26 julio de 2006. Disponível em: https:// econpapers.repec.org/paper/agsaaea06/21201.htm

Bleich, S.; Jarlenski, M.; Bell, C.; LaVeist, T. Health Inequalities: Trend, Progress and policy. Annual Review of Public Health, 33, 7-40, 2012. doi: 10.1146/annurev-publhealth-031811-124658

Bolsi, A.; Longhi, F.; Paolasso, P. El Norte Grande Argentino entre el progreso y la pobreza. Población y Sociedad, 12(1), 231-270, 2005.

Bradley, R.; Kelleher, K. Risk factors and outcomes for failure to thrive in low birth weight preterm infants. Pediatrics, 91(5), 941-948, 1993. Disponível em: http://pediatrics. aappublications.org/content/91/5/941

Brown, A. Bosques Nativos de Argentina. Trabajo presentado en el Congreso Forestal Mundial, 2009. Disponível em: http://www.ecopuerto.com/Bicentenario/informes/ BosquesNativosArgentina.pdf

Bucknall, J.; Kraus, C.; Pillai, P. Poverty and the Environment. Documento de trabajo del Banco Mundial, 2001. Disponível em: http://web.worldbank.org/archive/ website00346F/WEB/PDF/POVERTY_.PDF Acesso em: May, 2018.

Carter, M.; Little, P.; Mogues, T.; Negatu, W. Poverty Traps and Natural Disasters in Ethiopia and Honduras. World Development, 35(5), 835-856, 2007. doi: 10.1016/j. worlddev.2006.09.010 
Cernea, M. Poverty risks from population displacement in water resources development. Harvard Institute for International Development, trabajo de discusión, 1990. Disponível em: https://www.cabdirect.org/cabdirect/abstract/19916712675

Clarke, R. Global Environment Outlook 2000. Londres: Earthscan Publications, 1999.

Clichevsky, N. Pobreza y políticas urbano ambientales en Argentina. Santiago de Chile: Serie Medio Ambiente y Desarrollo CEPAL, 2002.

Comisión Mundial sobre Medio Ambiente y Desarrollo. Nuestro Futuro Común. Washington, 1987. Disponível em: http://www.un.org/es/comun/docs/?symbol=A/42/427

Dasgupta, S.; Deichmann, U.; Meisner C.; Wheeler, D. Where is the poverty-environment Nexus? Evidence from Cambodia, Lao PDR, and Vietnam. World Development, 33(4), 617-634, 2005. doi: 10.1016/j.worlddev.2004.10.003

Galvao, L; Finkelman, j.; Henao S. Environmental and social determinants of health. Organización Panamericana de la Salud, 2016. Disponível em: https://www.paho.org/ blogs/paltex/wp-content/uploads/2016/06/preliminares_english_finkelman.pdf Acesso em: jul. 2018.

Gertsakis, J.; Lewis, H. Sustainability and the Waste Management Hierarchy. EcoRecycle Victoria, Discussion paper, 2003. Disponível em: http://www.helenlewisresearch.com. au/wp-content/uploads/2014/05/TZW___Sustainability_ and the_Waste_Hierarchy_2003.pdfAcesso em: mar. 2018.

Getahun, K.; Biruktait, T.; Nardos, D. The Poverty-Environment Nexus in Depeloping countries: Evidence from Ethiopia: A systematic review. Asian Journal of Agricultural Extension, Economics and Sociology, 24(3), 1-13, 2018. doi: 10.9734/AJAEES/2018/39310

Gray, L.; Moseley, W. A geographical perspective on poverty-environment interactions. The Geographical Journal, 171(1), 9-23, 2005. doi: 10.1111/j.1475-4959.2005.00146.x

Gwatkin, D.; Guillot, M. The Burden of Disease among the Global Poor: Current situation, Future Trends and Implications for Strategy. Washington: Health, Nutrition and Population Series, 1999.

Hardoy, J.; Satterthwaite, D. Environmental Problems in an
Urbanizing World: Finding Solutions for Cities in Africa, Asia, and Latin America. Londres: Earthscan publications, 2001.

Johnson, D.; Ambrose, H.; Bassett, T.; Bowen, M.; Crummey, D.; Isaacson, J.; Johnson, D.; Lamb, P.; Saul, M.; WinterNelson, A. Meanings of Environmental terms. Journal of Environmental Quality, 26(3), 581-589, 1997. doi: 10.2134/ jeq1997.00472425002600030002x

Kurtz, H. Scale frames and counter-scale frames: constructing the problema of environmental justice. Political Geography, 22, 887-916, 2003. doi: 10.1016/j.polgeo.2003.09.001

Longhi, F.; Osatinsky, A. Estructura productiva, pobreza y problemas de empleo en las provincias pampeanas y norteñas de Argentina en los primeros años del siglo XXI. Cuadernos de Geografia, 26(1), 77-99, 2017. doi: 10.15446/ rcdg.v26n1.51011

Mink, S. Poverty, population and the environment. Banco Mundial, trabajo de discusión $\mathrm{N}^{\circ} 189,1993$. Disponível em: http://documents.worldbank.org/curated/ en/192051468741002945/Poverty-population-and-the-environment Acesso em: mar. 2018.

Mittermeir, R.; Da Fonseca, G.; Rylands, A.; Brandon, $\mathrm{K}$. A brief history of biodiversity conservation in Brazil. Conservation Biology, 19(3), 601-607, 2005. doi: 10.1111/j. 1523-1739.2005.00709.x

Moore, K. Frameworks for understandings the inter-generational transmission of poverty and well-being in developing countries. Documento de trabajo $\mathrm{N}^{\circ} 8$ CPRC, 2001. Disponível em: http://www.chronicpoverty.org/uploads/ publication_files/WP08_Moore.pdf Acesso em: mar. 2018.

Naciones Unidas. Memoria del Secretario General sobre la labor de la Organización. Nueva York, 2015. Disponível em: https://undocs.org/es/A/70/1 Acesso em: ago. 2018.

Narloch, U; Bangalore, M. The Multifaceted relationship between environmental risk and poverty: new insights from Vietnam. Environmental and Development Economics, 2018. doi: $10.1017 /$ S1355770X18000128

Nayak, P. Poverty and Environment degradation in rural India: A nexus. Trabajo presentado en la conferencia anual 
de NEEA, 2004. Disponível em: https://www.academia. edu/573615/Poverty_and_environmental_degradation_in_ rural_India_A_Nexus Acesso em: mar. 2018.

Nussbaumer, P.; Bazilian, M.; Modi, V. Measuring energy poverty: Focusing on what matters. Renewable and sustainable energy reviews, 16, 231-243, 2012. doi: 10.1016/j. rser.2011.07.150

Okwi, P. G.; Ndenge, P.; Kristjanson, M.; Arunga, A.; Notenbaert, A.; Omolo, A.; Henninger, D.; Kariuki, P. Geographic Determinants of Poverty in Kenya: A national and provincial analysis. ILRI Working Paper, 2006.

Olson, D.; Dinerstein, E. The Global 200: Priority Ecoregions for Global Conservation. Annals of the Missouri Botanical Garden, 89(2), 199-224, 2002. doi: 10.2307/3298564

Organización Mundial de la Salud. Preventing disease through healthy environments: Towards an estimate of the environmental burden of disease. 2006. Disponível em: http://www.who.int/quantifying_ehimpacts/publications/ preventingdisease.pdf Acesso em: may. 2018.

Organización Mundial de la Salud. World Rural Women's day. Declaración sobre polución dentro del hogar, 2004. Disponível em: http://www.who.int/mediacentre/news/ statements/2004/statement5/en/ Acesso em: may. 2018.

Pasanen, T.; Lakkala H.; Yliluoma, R.; Tuominen, V.; Jusi, S.; Luukkanen, J.; Kaivo-Oja, J. Poverty Environment Nexus in the LAO PDR: Analysis of household survey data. Development Policy Review, 35(3), 349-371, 2017. doi: 10.1111/dpr.12212

Pereira, G.; Nunes da Silva, M. Pobreza urbana e degradação ambiental: algumas reflexões sobre Curitiba, Brasil. Cuadernos de Vivienda y Urbanismo, 4(7), 122-135, 2011. Disponível em: http://revistas.javeriana.edu.co/index.php/ cvyu/article/view/5574/4401

Penttinen, A. Poverty and the Environment: Investment poverty and the role of assets in generating welfare for farmers household in the province of Herrera, Panama. Disertación académica en University of Helsinki, 2008. Disponível em: https://helda.helsinki.fi/bitstream/handle/10138/20911/ povertya.pdf?sequence=1 Acesso em: ago. 2018 .

Prakash, S. Poverty and Environment linkages in moun- tains and uplands: reflections on the poverty trap thesis. CREED Working Paper 12, 1997. Disponível em: http:// www.prem-online.org/archive/17/doc/creed12e.pdfAcesso em: may. 2018.

Santos, M. El Índice Multidimensional y trampas de pobreza en el Cono Sur. Revista Problemas del Desarrollo, 178(45), 89-112, 2014. Disponível em: www.revistas.unam.mx/ index.php/pde/article/download/47836/43015

Santos, M.; Zaratiegui, E. Pobreza en Argentina y Bahía Blanca: un enfoque de dominio estocástico. Período 20042016. En: Anales de la LII Reunión Anual de la Asociación Argentina de Economía Política. Bariloche, 15 de nov., 2017. Disponível em: http://www.aaep.org.ar/anales/works/ works2017/santos.pdf

Satterthwaite, D. The Links Between Poverty and the Environment in Urban Areas of Africa, Asia and Latin America. The Annals of the American Academy of Political and Social Science, 590(1), 73-92, 2003. doi: 10.1177/0002716203257095

Sherr, S.; Yadav, S. Land Degradation in the developing world: issues and policy options for 2020. International Food Policy Research Institute, Nota 44, 1997. Disponível em: http://ageconsearch.umn.edu/bitstream/16371/1/br44. pdf Acesso em: abr. 2018.

Sociedad Entomológica Argentina. Distribución geográfica del Dengue en Argentina. Boletín de la SEA, 2009. Disponível em: http://seargentina.myspecies.info/sites/ seargentina.myspecies.info/files/Bol.SEA20r_0.pdfAcesso em: may. 2018.

Sunkel, O. La dimensión ambiental en los Estilos de Desarrollo de América Latina. Libros de la CEPAL, 5, 1-136, 1980. Disponível em: http://archivo.cepal.org/pdfs/1980/ S8000002.pdf

Thiry, G.; Alkire, S.; Schleicher, J. Incorporating environmental and natural resources whitin analyses of multidimensional poverty. OPHI Research in Progress 50a., 2018. Disponível em: https://ophi.org.uk/incorporating-environmental-and-natural-resources-within-analyses-of-multidimensional-poverty/

Twesigye C. Poverty-Environment Indicators and Strategy for Monitoring them within the Framework of the EDPRS. 
Ruanda, Rwanda Envornmental Management Authority, 2007. Disponível em: https://www.unpei.org/sites/default/ files/e_library_documents/Rwanda-PE-Indicators.pdf

Vedeld, P.; Angelsen, A.; Bojo, J.; Sjaastad, E.; Kabugabe, B. Forest Environmental Incomes and the rural poor. Forest Policy and Economics, 9(7), 869-879, 2007.

World Wild Fund. Developing and appliyng poverty-environment indicators, 2004. Disponível em: http://www. portalces.org/sites/default/files/migrated/docs/1130.pdf Acesso em: abr. 2018. 